\title{
Solving system of partial differential equations using varia- tional iteration method with He's polynomials
}

\author{
Muhammad Nadeemª, Shao-Wen Yao ${ }^{\mathrm{b}, *}$ \\ a School of Mathematical Sciences, Dalian University of Technology, Dalian 116024, China. \\ ${ }^{b}$ School of Mathematics and Information Science, Henan Polytechnic University, Jiaozuo 454000, China.
}

\begin{abstract}
In the present work, variational iteration method with He's polynomials (VIMHP) is widely proposed to elucidate the linear and nonlinear system of partial differential equations. In the proposed method, variational iteration method is coupled with homotopy perturbation methods using He's polynomials to handle the nonlinear terms. We emphasize the efficiency of this approach by solving two appropriate examples. The significant results for solving the linear and nonlinear coupled system of equations demonstrate the superiority and competence of this approach. The proposed method finds the solution without any restrictive assumptions, discretization, and linearization.
\end{abstract}

Keywords: Coupled pseudo-parabolic equation, coupled Burgers equation, Lagrange multiplier, He's polynomials.

2010 MSC: 35K70, 35A20, 35F50.

(C)2019 All rights reserved.

\section{Introduction}

Linear and non-linear differential equations can model many phenomena in different fields of science and engineering in order to present their behaviors and effects by mathematical concepts. Most of the equations do not have analytical solution which can be handled by semi-analytical or numerical methods. In order to obtain exact solution of nonlinear differential equations, semi-analytical methods such as the variational Iteration method (VIM) and homotopy perturbation method (HPM) are considered. Variational iteration method was first proposed by the Chinese mathematician Ji-Huan He [7, 8]. It does not require a small parameter which has a significant advantage to provide an analytical solution for a wide range of linear and nonlinear problems in applied sciences. Later, Abdou and Soliman [1] showed the significant results by using variational iteration method for solving burger's and coupled burger's equations. Nadeem at el. [16] presented the application of variational iteration method for solving nonhomogeneous Cauchy Euler differential equations. The variational iteration method for solving linear and nonlinear system of PDEs is presented by [25]. Later, homotopy perturbation method [6, 9, 10] was developed to solve partial differential equation involving nonlinear terms. Khan and $\mathrm{Wu}$ [12] showed that homotopy perturbation transform method is an efficient tool for nonlinear equations using He's polynomials. The homotopy perturbation method is used by Nourazar et al. [18-20] in order to obtain

\footnotetext{
*Corresponding author

Email address: yaoshaowen@hpu.edu.cn (Shao-Wen Yao)
}

doi: 10.22436/jmcs.019.03.07

Received: 2019-04-26 Revised: 2019-05-03 Accepted: 2019-05-07 
exact solution of nonlinear differential equations. Recently, Nadeem at el. [17] coupled Laplace transform with He's polynomials for solving fourth-order parabolic partial differential equation with variable coefficients. Anjum and He [4] suggested an easier identification process of Lagrange multiplier by coupling of Laplace transform and variational iteration method for solving non-linear oscillator equation. MohyudDin and Yildirim [14] presented the variational iteration method for delay differential equations using He's polynomials.

The aim of this article is to extend the application of the variational iteration method to solve linear and nonlinear system of partial differential equations such as the system of coupled pseudo-parabolic and coupled Burgers equations. The present method is also valid for differential equations with fractal derivatives [11, 13, 21-24].

\section{Variational iteration method}

To clarify the VIM, we begin by considering a differential equation in the formal form $[2,3,15]$ :

$$
\mathrm{Lu}(x, \mathrm{t})+\mathrm{Nu}(x, \mathrm{t})=\mathrm{g}(\mathrm{x}, \mathrm{t}),
$$

where $L$ and $N$ are linear and nonlinear operators respectively, and $g(x, t)$ is a known analytical function.

The VIM allows us to write a correct functional of the following type:

$$
u_{n+1}(x, t)=u_{n}(x, t)+\int_{0}^{t} \lambda(s)\left[\operatorname{Lu}_{n}(x, s)+N \tilde{u}_{n}(x, s)-g(x, s)\right] d s,
$$

where $\lambda$ is a general Lagrange's multiplier, which can be identified optimally via the variational theory and $\tilde{u}_{n}$ is a restricted for variation which means $\delta \tilde{u}_{n}=0$, yields the following Lagrange multipliers

$$
\lambda=-1 \text { for } m=1, \quad \lambda=s-t \text { for } m=2,
$$

and in general, for $m \geqslant 1$,

$$
\lambda=\frac{(-1)^{m}(s-t)^{m-1}}{(m-1) !} .
$$

Therefore, substituting (2.2) into functional (2.1) we obtain the following iteration formula,

$$
u_{n+1}(x, t)=u_{n}(x, t)+\int_{0}^{t} \frac{(-1)^{m}(s-t)^{m-1}}{(m-1) !}\left[\operatorname{Lu}(x, s)+N \tilde{u}_{n}(x, s)-g(x, s)\right] d s .
$$

Thus Eq. (2.3) is called as a correction functional. The successive approximation $u_{n+1}, n \geqslant 0$ of the solution $u$ will be readily obtained upon using the determined Lagrange multiplier and any selective function $u_{0}$. Consequently, the solution is given by

$$
u=\lim _{n \rightarrow \infty} u_{n} .
$$

\section{Basic idea of homotopy perturbation method}

To illustrate the basic concept of homotopy perturbation method, consider the following non-linear functional equation $[15,17]$

$$
A(u)-f(r)=0, \quad r \in \Omega,
$$

with boundary conditions

$$
B\left(u, \frac{\partial u}{\partial n}\right)=0, \quad r \in \Gamma,
$$

where $A$ is a general functional operator, $B$ is a boundary operator, $f(r)$ is a known analytic function, and 
$\Gamma$ is the boundary of the domain $\Omega$. The operator $A$ can generally be divided into two operators, $L$ and $\mathrm{N}$, where $\mathrm{L}$ is a linear and $\mathrm{N}$ being a nonlinear operator. Therefore, Eq. (3.1) can be written as follows

$$
L(u)+N(u)-f(r)=0 .
$$

Using the homotopy technique, we construct a homotopy $v(r, p): \Omega \times[0,1] \rightarrow \mathbb{R}$, which satisfies

$$
H(v, p)=(1-p)\left[L(v)-L\left(u_{0}\right)\right]+p[L(v)-N(v)-f(r)],
$$

or

$$
\mathrm{H}(v, p)=\mathrm{L}(v)-\mathrm{L}\left(\mathrm{u}_{0}\right)+\mathrm{pL}\left(\mathrm{u}_{0}\right)+\mathrm{p}[\mathrm{N}(v)-\mathrm{f}(\mathrm{r})]=0,
$$

where $p \in[0,1]$, is called homotopy parameter, and $u_{0}$ is an initial approximation for the solution of Eq. (3.1), which satisfies the boundary conditions. According to HPM, we can use $p$ as a small parameter, and assume that the solution of Eq. (3.2) can be written as a power series in $p$

$$
v=v_{0}+p v_{1}+p^{2} v_{2}+\cdots
$$

Considering $p=1$, the approximate solution of Eq. (3.1) will be obtained as follows

$$
u=\lim _{p \rightarrow 1} v=v_{0}+v_{1}+v_{2}+v_{3}+\cdots .
$$

Substituting (3.3) into (3.2) and equating the terms with identical powers of $p$, we can obtain a series of equations of the following form:

$$
\begin{array}{r}
p^{0}: v_{0}-f(x)=0 \\
p^{1}: v_{1}-H\left(v_{0}\right)=0 \\
p^{2}: v_{2}-H\left(v_{0}, v_{1}\right)=0 \\
p^{3}: v_{3}-H\left(v_{0}, v_{1}, v_{2}\right)=0
\end{array}
$$

where $\mathrm{H}\left(v_{0}, v_{1}, v_{2}, \ldots, v_{\mathrm{j}}\right)$ depend upon $v_{0}, v_{1}, v_{2}, \ldots, v_{j}$ and are the so-called He's polynomials, which can be calculated by using the formula

$$
\mathrm{H}\left(v_{0}, v_{1}, v_{2}, \ldots, v_{j}\right)=\left.\frac{1}{j !} \frac{\partial^{j}}{\partial p^{j}} \mathrm{~N}\left(\sum_{i=0}^{j} v_{i} p^{i}\right)\right|_{p=0} .
$$

It is obvious that the system of nonlinear equations in (3.4) is easy to solve, and the components $v_{i}, i \geqslant 0$ of the homotopy perturbation method can be completely determined, and the series solutions are thus entirely determined.

\section{Variational iteration method with He's polynomials}

In this section, we highlight briefly the main point of the VIMHP, where more details can be found in $[5,26]$. We consider the following equation:

$$
\operatorname{Lu}(x, t)+N u(x, t)=g(x, t) .
$$

The VIM allows us to write a correct functional of the following type:

$$
u_{n+1}(x, t)=u_{n}(x, t)+\int_{0}^{t} \lambda(s)\left[L u_{n}(x, s)+N \tilde{u}_{n}(x, s)-g(x, s)\right] d s,
$$


where $\lambda$ is a general Lagrange's multiplier. Now, by using the homotopy perturbation method $[6,9,10]$, we can construct an equation as follows:

$$
\sum_{n=0}^{\infty} p^{n} u_{n}(x, t)=u_{0}(x, t)+p \int_{0}^{t} \lambda(s)\left[N\left(\sum_{n=0}^{\infty} p^{n} u_{n}(x, s)\right)-g(x, s)\right] d s .
$$

As it is seen, the procedure is constructed by coupling of VIM and HPM methods. A comparison of like powers of $p$ gives solutions of various orders. By equating the terms of (4.1) with identical powers of $p$, and taking the limit as $p$ tends to 1 , we obtain

$$
u(x, t)=\lim _{p \rightarrow 1} \sum_{n=0}^{\infty} p^{n} u_{n}(x, t)=u_{0}(x, t)+u_{1}(x, t)+u_{2}(x, t)+\cdots
$$

\section{Numerical applications}

In this section, we present the analytical solution of two examples, namely, coupled pseudo-parabolic equation and coupled Burgers equation. The significant results show the accuracy and the effectiveness of the present method.

\subsection{Example 1}

Consider the following homogeneous form of a coupled pseudo-parabolic equation

$$
\frac{\partial u}{\partial t}-\frac{1}{x} \frac{\partial}{\partial x}\left(x \frac{\partial u}{\partial x}\right)-\frac{1}{x} \frac{\partial^{2}}{\partial x \partial t}\left(x \frac{\partial u}{\partial x}\right)+v=0, \quad \frac{\partial v}{\partial t}-\frac{1}{x} \frac{\partial}{\partial x}\left(x \frac{\partial v}{\partial x}\right)-\frac{1}{x} \frac{\partial^{2}}{\partial x \partial t}\left(x \frac{\partial v}{\partial x}\right)+u=0,
$$

with initial condition

$$
u(x, 0)=x^{2}, \quad v(x, 0)=x^{2} .
$$

The correct functional for system of Eq. (5.1) is given as

$$
\begin{aligned}
& u_{n+1}(x, t)=u_{n}(x, t)+\int_{0}^{t} \lambda_{1}(s)\left[\frac{\partial u_{n}}{\partial s}-\frac{1}{x} \frac{\partial}{\partial x}\left(x \frac{\partial \tilde{u}_{n}}{\partial x}\right)-\frac{1}{x} \frac{\partial^{2}}{\partial x \partial s}\left(x \frac{\partial \tilde{u}_{n}}{\partial x}\right)+v_{n}\right] d s, \\
& v_{n+1}(x, t)=v_{n}(x, t)+\int_{0}^{t} \lambda_{2}(s)\left[\frac{\partial v_{n}}{\partial s}-\frac{1}{x} \frac{\partial}{\partial x}\left(x \frac{\partial \tilde{v}_{n}}{\partial x}\right)-\frac{1}{x} \frac{\partial^{2}}{\partial x \partial s}\left(x \frac{\partial \tilde{v}_{n}}{\partial x}\right)+u_{n}\right] d s .
\end{aligned}
$$

This yields the stationary conditions

$$
1+\lambda_{1}(s)=0, \quad \lambda_{1}^{\prime}(s=t)=0, \quad 1+\lambda_{2}(s)=0, \quad \lambda_{2}^{\prime}(s=t)=0 .
$$

The Lagrange multipliers can be identified as follows:

$$
\lambda_{1}(s)=\lambda_{2}(s)=-1 .
$$

Substituting these values of the Lagrange multipliers into the functionals (5.2) and (5.3), gives the iteration formulas

$$
u_{n+1}(x, t)=u_{n}(x, t)-\int_{0}^{t}\left[\frac{\partial u_{n}}{\partial s}-\frac{1}{x} \frac{\partial}{\partial x}\left(x \frac{\partial u_{n}}{\partial x}\right)-\frac{1}{x} \frac{\partial^{2}}{\partial x \partial s}\left(x \frac{\partial u_{n}}{\partial x}\right)+v_{n}\right] d s
$$




$$
v_{n+1}(x, t)=v_{n}(x, t)-\int_{0}^{t}\left[\frac{\partial v_{n}}{\partial s}-\frac{1}{x} \frac{\partial}{\partial x}\left(x \frac{\partial v_{n}}{\partial x}\right)-\frac{1}{x} \frac{\partial^{2}}{\partial x \partial s}\left(x \frac{\partial v_{n}}{\partial x}\right)+u_{n}\right] d s
$$

Applying the variational homotopy perturbation method on (5.4) and (5.5), respectively, we have:

$$
\begin{aligned}
& \sum_{n=0}^{\infty} p^{n} u_{n}(x, t)=u_{0}(x, t)+p \int_{0}^{t}\left[\frac{1}{x} \frac{\partial}{\partial x}\left(x \sum_{n=0}^{\infty} p^{n} \frac{\partial u_{n}}{\partial x}\right)+\frac{1}{x} \frac{\partial^{2}}{\partial x \partial s}\left(x \sum_{n=0}^{\infty} p^{n} \frac{\partial u_{n}}{\partial x}\right)-\sum_{n=0}^{\infty} p^{n} v_{n}\right] d s \\
& \sum_{n=0}^{\infty} p^{n} v_{n}(x, t)=v_{0}(x, t)+p \int_{0}^{t}\left[\frac{1}{x} \frac{\partial}{\partial x}\left(x \sum_{n=0}^{\infty} p^{n} \frac{\partial v_{n}}{\partial x}\right)+\frac{1}{x} \frac{\partial^{2}}{\partial x \partial s}\left(x \sum_{n=0}^{\infty} p^{n} \frac{\partial v_{n}}{\partial x}\right)-\sum_{n=0}^{\infty} p^{n} u_{n}\right] d s .
\end{aligned}
$$

We can select $u_{0}(x, t)=x^{2}, v_{0}(x, t)=x^{2}$ by using the given initial values. Accordingly, we obtain the following successive approximations by comparing the coefficient of like powers of $p$,

$$
\begin{aligned}
& p^{0}: u_{0}(x, t)=x^{2} \\
& p^{0}: v_{0}(x, t)=x^{2} \\
& p^{1}: u_{1}(x, t)=\int_{0}^{t}\left[\frac{1}{x} \frac{\partial}{\partial x}\left(x \frac{\partial u_{0}}{\partial x}\right)+\frac{1}{x} \frac{\partial^{2}}{\partial x \partial s}\left(x \frac{\partial u_{0}}{\partial x}\right)-v_{0}\right] d s=4 t-x^{2} t \\
& p^{1}: v_{1}(x, t)=\int_{0}^{t}\left[\frac{1}{x} \frac{\partial}{\partial x}\left(x \frac{\partial v_{0}}{\partial x}\right)+\frac{1}{x} \frac{\partial^{2}}{\partial x \partial s}\left(x \frac{\partial v_{0}}{\partial x}\right)-u_{0}\right] d s=4 t-x^{2} t \\
& p^{2}: u_{2}(x, t)=\int_{0}^{t}\left[\frac{1}{x} \frac{\partial}{\partial x}\left(x \frac{\partial u_{1}}{\partial x}\right)+\frac{1}{x} \frac{\partial^{2}}{\partial x \partial s}\left(x \frac{\partial u_{1}}{\partial x}\right)-v_{1}\right] d s=-4 t^{2}-4 t+\frac{x^{2} t^{2}}{2} \\
& p^{2}: v_{2}(x, t)=\int_{0}^{t}\left[\frac{1}{x} \frac{\partial}{\partial x}\left(x \frac{\partial v_{1}}{\partial x}\right)+\frac{1}{x} \frac{\partial^{2}}{\partial x \partial s}\left(x \frac{\partial v_{1}}{\partial x}\right)-u_{1}\right] d s=-4 t^{2}-4 t+\frac{x^{2} t^{2}}{2} \\
& p^{3}: u_{3}(x, t)=\int_{0}^{t}\left[\frac{1}{x} \frac{\partial}{\partial x}\left(x \frac{\partial u_{2}}{\partial x}\right)+\frac{1}{x} \frac{\partial^{2}}{\partial x \partial s}\left(x \frac{\partial u_{2}}{\partial x}\right)-v_{2}\right] d s=2 t^{3}+4 t^{2}-\frac{x^{2} t^{3}}{6} \\
& p^{3}: v_{3}(x, t)=\int_{0}^{t}\left[\frac{1}{x} \frac{\partial}{\partial x}\left(x \frac{\partial v_{2}}{\partial x}\right)+\frac{1}{x} \frac{\partial^{2}}{\partial x \partial s}\left(x \frac{\partial v_{2}}{\partial x}\right)-u_{2}\right] d s=2 t^{3}+4 t^{2}-\frac{x^{2} t^{3}}{6} \\
& p^{4}: v_{4}(x, t)=\int_{0}^{t}\left[\frac{1}{x} \frac{\partial}{\partial x}\left(x \frac{\partial v_{3}}{\partial x}\right)+\frac{1}{x} \frac{\partial^{2}}{\partial x \partial s}\left(x \frac{\partial v_{3}}{\partial x}\right)-u_{3}\right] d s=-\frac{2}{3} t^{4}-2 t^{3}+\frac{x^{2} t^{4}}{24} \\
& p^{4}: u_{4}(x, t)=\int_{0}^{t}\left[\frac{1}{x} \frac{\partial}{\partial x}\left(x \frac{\partial u_{3}}{\partial x}\right)+\frac{1}{x} \frac{\partial^{2}}{\partial x \partial s}\left(x \frac{\partial u_{3}}{\partial x}\right)-v_{3}\right] d s=-\frac{2}{3} t^{4}-2 t^{3}+\frac{x^{2} t^{4}}{24}
\end{aligned}
$$

The series solutions are therefore given by

$$
u(x, t)=u_{0}+u_{1}+u_{2}+u_{3}+\cdots=\left(1-t+\frac{t^{2}}{2 !}-\frac{t^{3}}{3 !}+\frac{t^{4}}{4 !}-\cdots\right) x^{2},
$$




$$
v(x, t)=v_{0}+v_{1}+v_{2}+v_{3}+\cdots=\left(1-t+\frac{t^{2}}{2 !}-\frac{t^{3}}{3 !}+\frac{t^{4}}{4 !}-\cdots\right) x^{2},
$$

and hence the exact solution is

$$
u(x, t)=x^{2} e^{-t}, \quad v(x, t)=x^{2} e^{-t}
$$

\subsection{Example 2}

Consider the following one-dimensional coupled Burgers equation:

$$
\begin{gathered}
\frac{\partial u}{\partial t}-\frac{\partial^{2} u}{\partial x^{2}}-2 u \frac{\partial u}{\partial x}+\frac{\partial}{\partial x}(u v)=0 \\
\frac{\partial v}{\partial t}-\frac{\partial^{2} v}{\partial x^{2}}-2 v \frac{\partial v}{\partial x}+\frac{\partial}{\partial x}(u v)=0
\end{gathered}
$$

with the initial conditions:

$$
u(x, 0)=\cos x, \quad v(x, 0)=\sin x .
$$

The correct functional for system of Eq. (5.6) is given as

$$
\begin{aligned}
& u_{n+1}(x, t)=u_{n}(x, t)+\int_{0}^{t} \lambda_{3}(s)\left[\frac{\partial u_{n}}{\partial s}-\frac{\partial^{2} \tilde{u}_{n}}{\partial x^{2}}-2 \tilde{u}_{n} \frac{\partial \tilde{u}_{n}}{\partial x}+\frac{\partial}{\partial x}\left(\tilde{u}_{n} \tilde{v}_{n}\right)\right] d s, \\
& v_{n+1}(x, t)=v_{n}(x, t)+\int_{0}^{t} \lambda_{4}(s)\left[\frac{\partial v_{n}}{\partial s}-\frac{\partial^{2} \tilde{v}_{n}}{\partial x^{2}}-2 \tilde{v}_{n} \frac{\partial \tilde{v}_{n}}{\partial x}+\frac{\partial}{\partial x}\left(\tilde{u}_{n} \tilde{v}_{n}\right)\right] d s .
\end{aligned}
$$

This yields the stationary conditions

$$
1+\lambda_{3}(s)=0, \quad \lambda_{3}^{\prime}(s=t)=0, \quad 1+\lambda_{4}(s)=0, \quad \lambda_{4}^{\prime}(s=t)=0 .
$$

The Lagrange multipliers can be identified as follows:

$$
\lambda_{3}(s)=\lambda_{4}(s)=-1 .
$$

Substituting these values of the Lagrange multipliers into the functionals (5.7) and (5.8) gives the iteration formulas

$$
\begin{aligned}
& u_{n+1}(x, t)=u_{n}(x, t)-\int_{0}^{t} \lambda_{3}(s)\left[\frac{\partial u_{n}}{\partial s}-\frac{\partial^{2} u_{n}}{\partial x^{2}}-2 u_{n} \frac{\partial u_{n}}{\partial x}+\frac{\partial}{\partial x}\left(u_{n} v_{n}\right)\right] d s \\
& v_{n+1}(x, t)=v_{n}(x, t)-\int_{0}^{t} \lambda_{4}(s)\left[\frac{\partial v_{n}}{\partial s}-\frac{\partial^{2} v_{n}}{\partial x^{2}}-2 v_{n} \frac{\partial v_{n}}{\partial x}+\frac{\partial}{\partial x}\left(u_{n} v_{n}\right)\right] d s
\end{aligned}
$$

Applying the variational homotopy perturbation method on (5.9) and (5.10) respectively, we have:

$$
\begin{aligned}
& \sum_{n=0}^{\infty} p^{n} u_{n}(x, t)=u_{0}(x, t)+p \int_{0}^{t}\left[\sum_{n=0}^{\infty} p^{n} \frac{\partial^{2} u_{n}}{\partial x^{2}}+2\left(\sum_{n=0}^{\infty} p^{n} u_{n}\right)\left(\sum_{n=0}^{\infty} p^{n} \frac{\partial u_{n}}{\partial x}\right)-\sum_{n=0}^{\infty} p^{n} \frac{\partial}{\partial x}\left(u_{n} v_{n}\right)\right] d s, \\
& \sum_{n=0}^{\infty} p^{n} v_{n}(x, t)=v_{0}(x, t)+p \int_{0}^{t}\left[\sum_{n=0}^{\infty} p^{n} \frac{\partial^{2} v_{n}}{\partial x^{2}}+2\left(\sum_{n=0}^{\infty} p^{n} v_{n}\right)\left(\sum_{n=0}^{\infty} p^{n} \frac{\partial v_{n}}{\partial x}\right)-\sum_{n=0}^{\infty} p^{n} \frac{\partial}{\partial x}\left(u_{n} v_{n}\right)\right] d s .
\end{aligned}
$$


We can select $u_{0}(x, t)=\cos x, v_{0}(x, t)=\cos x$ by using the given initial values. Accordingly, we obtain the following successive approximations by comparing the coefficient of like powers of $p$,

$$
\begin{aligned}
& p^{0}: u_{0}(x, t)=\cos x, \\
& p^{0}: v_{0}(x, t)=\cos x \text {, } \\
& p^{1}: u_{1}(x, t)=\int_{0}^{t}\left[\frac{\partial^{2} u_{0}}{\partial x^{2}}+2 u_{0} \frac{\partial u_{0}}{\partial x}-\frac{\partial}{\partial x}\left(u_{0} v_{0}\right] d s=-t \cos x\right. \\
& p^{1}: v_{1}(x, t)=\int_{0}^{t}\left[\frac{\partial^{2} v_{0}}{\partial x^{2}}+2 v_{0} \frac{\partial v_{0}}{\partial x}-\frac{\partial}{\partial x}\left(u_{0} v_{0}\right] d s=-t \cos x\right. \\
& p^{2}: u_{2}(x, t)=\int_{0}^{t}\left[\frac{\partial^{2} u_{1}}{\partial x^{2}}+2 u_{0} \frac{\partial u_{1}}{\partial x}+2 u_{1} \frac{\partial u_{0}}{\partial x}-\frac{\partial}{\partial x}\left(u_{0} v_{1}+u_{1} v_{0}\right)\right] d s=\frac{t^{2}}{2} \cos x \\
& p^{2}: v_{2}(x, t)=\int_{0}^{t}\left[\frac{\partial^{2} v_{1}}{\partial x^{2}}+2 v_{0} \frac{\partial v_{1}}{\partial x}+2 v_{1} \frac{\partial v_{0}}{\partial x}-\frac{\partial}{\partial x}\left(u_{0} v_{1}+u_{1} v_{0}\right)\right] d s=\frac{t^{2}}{2} \cos x \\
& p^{3}: u_{3}(x, t)=\int_{0}^{t}\left[\frac{\partial^{2} u_{2}}{\partial x^{2}}+2 u_{0} \frac{\partial u_{2}}{\partial x}+2 u_{1} \frac{\partial u_{1}}{\partial x}+2 u_{2} \frac{\partial u_{0}}{\partial x}-\frac{\partial}{\partial x}\left(u_{0} v_{2}+u_{1} v_{1}+u_{2} v_{0}\right)\right] d s=-\frac{t^{3}}{6} \cos x \\
& p^{3}: v_{3}(x, t)=\int_{0}^{t}\left[\frac{\partial^{2} v_{2}}{\partial x^{2}}+2 v_{0} \frac{\partial v_{2}}{\partial x}+2 v_{1} \frac{\partial v_{1}}{\partial x}+2 v_{2} \frac{\partial v_{0}}{\partial x}-\frac{\partial}{\partial x}\left(u_{0} v_{2}+u_{1} v_{1}+u_{2} v_{0}\right)\right] d s=-\frac{t^{3}}{6} \cos x, \\
& p^{4}: u_{4}(x, t)=\int_{0}^{t}\left[\frac{\partial^{2} u_{3}}{\partial x^{2}}+2 u_{0} \frac{\partial u_{3}}{\partial x}+2 u_{1} \frac{\partial u_{2}}{\partial x}+2 u_{2} \frac{\partial u_{1}}{\partial x}+2 u_{3} \frac{\partial u_{0}}{\partial x}-\frac{\partial}{\partial x}\left(u_{0} v_{3}+u_{1} v_{2}+u_{2} v_{1}+u_{3} v_{0}\right)\right] d s \\
& =\frac{\mathrm{t}^{4}}{24} \cos x, \\
& p^{4}: v_{4}(x, t)=\int_{0}^{t}\left[\frac{\partial^{2} v_{3}}{\partial x^{2}}+2 v_{0} \frac{\partial v_{3}}{\partial x}+2 v_{1} \frac{\partial v_{2}}{\partial x}+2 v_{2} \frac{\partial v_{1}}{\partial x}+2 v_{3} \frac{\partial v_{0}}{\partial x}-\frac{\partial}{\partial x}\left(u_{0} v_{3}+u_{1} v_{2}+u_{2} v_{1}+u_{3} v_{0}\right)\right] d s \\
& =\frac{\mathrm{t}^{4}}{24} \cos x,
\end{aligned}
$$

The series solutions are therefore given by

$$
\begin{aligned}
& u(x, t)=u_{0}+u_{1}+u_{2}+u_{3}+\cdots=\left(1-t+\frac{t^{2}}{2 !}-\frac{t^{3}}{3 !}+\frac{t^{4}}{4 !}-\cdots\right) \cos x, \\
& v(x, t)=v_{0}+v_{1}+v_{2}+v_{3}+\cdots=\left(1-t+\frac{t^{2}}{2 !}-\frac{t^{3}}{3 !}+\frac{t^{4}}{4 !}-\cdots\right) \cos x,
\end{aligned}
$$

and hence the exact solution becomes

$$
u(x, t)=e^{-t} \cos x, \quad v(x, t)=e^{-t} \cos x .
$$

\section{Conclusion}

In this paper, an analytical method called the variational iteration method with He's polynomials (VIMHP) is successfully applied to coupled pseudo-parabolic and coupled Burgers equations together 
with the initial conditions. In the present work, first, we use the variational iteration method to identify the Lagrange multiplier and then homotopy perturbation method with He's polynomials is employed to compute nonlinear terms. The flexibility and high accuracy of the analytical method is successfully demonstrated by illustrated examples. It is worth pointing out that the VIMHP presents rapid convergence towards exact solutions. Finally, we concluded that the proposed scheme is very powerful, efficient, and reliable in finding the analytical solutions as well as valid for other nonlinear case, especially for fractal calculus and fractional calculus for a wider class of linear and nonlinear system of partial differential equations.

\section{Acknowledgment}

Authors are special thanks to Prof. Dr. Ji-Huan He to improve this paper as well as Editor in Chief and anonymous referees for the valuable comments and suggestions. This work is supported by National Natural Science Foundation of China (No. 71601072).

\section{References}

[1] M. A. Abdou, A. A. Soliman, Variational iteration method for solving Burger's and coupled Burger's equations, J. Comput. Appl. Math., 181 (2005), 245-251. 1

[2] M. Al-Jawary, An efficientAn efficient iterative method for solving the Fokker-Planck equation, Results phys., 6 (2016), 985-991. 2

[3] M. A. Al-Jawary, G. H. Radhi, J. Ravnik, Daftardar-Jafari method for solving nonlinear thin film flow problem, Arab J. Basic Appl. Sci., 25 (2018), 20-27. 2

[4] N. Anjum, J.-H. He, Laplace transform: Making the variational iteration method easier, Appl. Math. Lett., 92 (2019), 134-138. 1

[5] A. Daga, V. H. Pradhan, Variational homotopy perturbation method for solving nonlinear reaction-diffusion convection problems, Int. J. Adv. Engg. Res. Studies, 2 (2013), 11-14. 4

[6] J.-H. He, Homotopy perturbation technique, Comput. Methods Appl. Mech. Engrg., 178 (1999), 257-262. 1, 4

[7] J.-H. He, Variational iteration method-a kind of non-linear analytical technique: some examples, Internat. J. Non-Linear Mech., 34 (1999), 699-708. 1

[8] J.-H. He, Variational iteration method for autonomous ordinary differential systems, Appl. Math. Comput., 114 (2000), 115-123. 1

[9] J.-H. He, Homotopy perturbation method: a new nonlinear analytical technique, Appl. Math. Comput., 135 (2003), 73-79. 1,4

[10] J.-H. He, Application of homotopy perturbation method to nonlinear wave equations, Chaos Solitons Fractals, 26 (2005), 695-700. 1, 4

[11] J.-H. He, Fractal calculus and its geometrical explanation, Results Phys., 10 (2018), 272-276. 1

[12] Y. Khan, Q. B. Wu, Homotopy perturbation transform method for nonlinear equations using He's polynomials, Comput. Math. Appl., 61 (2011), 1963-1967. 1

[13] X.-X. Li, D. Tian, C.-H. He, J.-H. He, A fractal modification of the surface coverage model for an electrochemical arsenic sensor, Electrochimica Acta, 296 (2019), 491-493. 1

[14] S. T. Mohyud-Din, A. Yildirim, Variational iteration method for delay differential equations using He's polynomials, Z. Naturforsch. A, 65 (2010), 1045-1048. 1

[15] M. Nadeem, F. Li, He-Laplace method for nonlinear vibration systems and nonlinear wave equations, J. Low Freq. Noise Vib. Active Contr., 2019 (2019), 15 pages. 2, 3

[16] M. Nadeem, F. Li, H. Ahmad, He's variational iteration method for solving non-homogeneous Cauchy Euler differential equations, Nonlinear Sci. Lett. A Math. Phys. Mech., 9 (2018), 231-237. 1

[17] M. Nadeem, F. Li, H. Ahmad, Modified Laplace variational iteration method for solving fourth-order parabolic partial differential equation with variable coefficients, Comput. Math. Appl., 2019 (2019), 10 pages. 1, 3

[18] S. S. Nourazar, M. Soori, A. Nazari-Golshan, On the exact solution of Newell-Whitehead-Segel equation using the homotopy perturbation method, Aust. J. Basic Appl. Sci., 5 (2011), 1400-1411. 1

[19] S. S. Nourazar, M. Soori, A. Nazari-Golshan, On the exact solution of Burgers-Huxley equation using the homotopy perturbation method, J. Appl. Math. Phy., 3 (2015), 285-294.

[20] S. S. Nourazar, M. Soori, A. Nazari-Golshan, On the homotopy perturbation method for the exact solution of FitzhughNagumo equation, Int. J. Math. Comput., 27 (2015), 32-43. 1

[21] Z.-F. Ren, G.-F. Hu, He's frequency-amplitude formulation with average residuals for nonlinear oscillators, J. Low Freq. Noise Vib. Active Contr., 2018 (2018), 10 pages. 1 
[22] Y. Wang, J.-Y. An, Amplitude-frequency relationship to a fractional Duffing oscillator arising in microphysics and tsunami motion, J. Low Freq. Noise Vib. Active Contr., 2018 (2018), 5 pages.

[23] Y. Wang, Q. Deng, Fractal derivative model for tsunamt travelling, Fractals, 27 (2019), 3 pages.

[24] Q. Wang, X. Shi, J.-H. He, Z. B. Li, Fractal calculus and its application to explanation of biomechanism of polar bear hairs, Fractals, 26 (2018), 5 pages. 1

[25] A.-M. Wazwaz, The variational iteration method for solving linear and nonlinear systems of PDEs, Comput. Math. Appl., 54 (2007), 895-902. 1

[26] M. Zellal, K. Belghaba, An accurate algorithm for solving biological population model by the variational iteration method using He's polynomials, Arab J. Basic Appl. Sci., 25 (2018), 142-149. 4 